TITLE:

\title{
Variational Construction of Orbits Realizing Symbolic Sequences in the Planar Sitnikov Problem
}

\author{
$\operatorname{AUTHOR(S):~}$
}

Shibayama, Mitsuru

\section{CITATION:}

Shibayama, Mitsuru. Variational Construction of Orbits Realizing Symbolic Sequences in the Planar Sitnikov Problem. Regular and Chaotic Dynamics 2019, 24(2): 202-211

\section{ISSUE DATE:}

2019-03

URL:

http://hdl.handle.net/2433/266301

\section{RIGHT:}

This is a post-peer-review, pre-copyedit version of an article published in 'Regular and Chaotic Dynamics'. The final authenticated version is available online at: https://doi.org/10.1134/\$1560354719020060.; The full-text file will be made open to the public on 10 April 2020 in accordance with publisher's 'Terms and Conditions for Self-Archiving'.; This is not the published version. Please cite only the published version.この論文は出版社版でありません。引用の際には出版社 版をご確認ご利用ください。 


\title{
Variational construction of orbits realizing sequences in the planar Sitnikov problem
}

\author{
Mitsuru Shibayama*
}

\begin{abstract}
By using the variational method, we show the existence of various kinds of solutions in the planar Sitnikov problem. For a given symbolic sequence, we show the existence of orbit realizing it. We also prove the existence of periodic orbits.
\end{abstract}

\section{Introduction}

Consider the three-body problem which is governed by the following ODEs:

$$
\begin{aligned}
& \ddot{q}_{1}=-m_{2} \frac{q_{1}-q_{2}}{\left|q_{1}-q_{2}\right|^{3}}-m_{3} \frac{q_{1}-q_{3}}{\left|q_{1}-q_{3}\right|^{3}} \\
& \ddot{q}_{2}=-m_{1} \frac{q_{2}-q_{1}}{\left|q_{2}-q_{1}\right|^{3}}-m_{3} \frac{q_{2}-q_{3}}{\left|q_{2}-q_{3}\right|^{3}} \\
& \ddot{q}_{3}=-m_{1} \frac{q_{3}-q_{1}}{\left|q_{3}-q_{1}\right|^{3}}-m_{2} \frac{q_{3}-q_{2}}{\left|q_{3}-q_{2}\right|^{3}} .
\end{aligned}
$$

Here $q_{k} \in \mathbb{R}^{3}$ and $m_{k} \geq 0$ for $k=1,2,3$ represent positions and masses.

Sitnikov [5] considered a special case of the three-body problem in which two masses are equal and the other is zero, and in which the massless particle moves on the $z$-axis and the other two masses move along elliptic curves on $x y$ plane which are periodic orbits of the Kepler problem(Figure 1). That model is called the Sitnikov problem. This model is famous for the subsystem in which oscillatory orbits are first proven to exist.

On the other hand, Chenciner \& Montogomery [1] proved the existence of an eight-shaped periodic solution in the planar three-body problem with equal masses by using the variational method. Since then, a number of periodic and quasi-periodic solutions have been found as minimizers of variational formulation of the $N$-body problem in various different settings.

* Department of Applied Mathematics and Physics, Graduate School of Informatics, Kyoto University, Yoshida-Honmachi, Sakyo-ku Kyoto 606-8501, Japan shibayama@amp.i.kyoto-u.ac.jp 


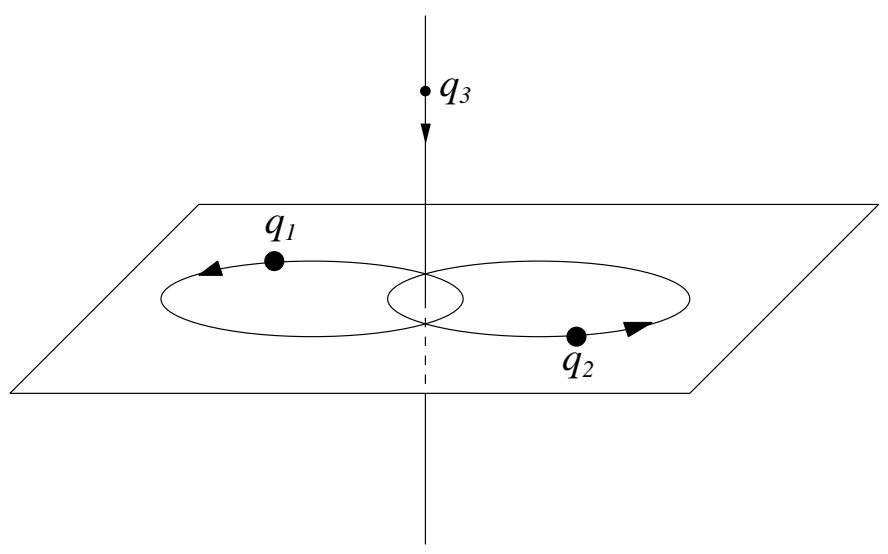

Figure 1: Sitnikov problem

In this paper we study the planar Sitnikov problem. Let $m_{1}=m_{2}=1>0$ and $m_{3}=0$ for (1). The equations become

$$
\begin{array}{lll}
\ddot{q}_{1} & =-\frac{q_{1}-q_{2}}{\left|q_{1}-q_{2}\right|^{3}} & \\
\ddot{q}_{2} & =-\frac{q_{2}-q_{1}}{\left|q_{2}-q_{1}\right|^{3}} & \left(q_{1}, q_{2}, q_{3} \in \mathbb{R}^{2}\right) \\
\ddot{q}_{3} & =-\frac{q_{3}-q_{1}}{\left|q_{3}-q_{1}\right|^{3}}-\frac{q_{3}-q_{2}}{\left|q_{3}-q_{2}\right|^{3}} . &
\end{array}
$$

The motion of $q_{1}$ and $q_{2}$ is governed by the two-body problem.

There is a subproblem such that $q_{1}$ and $q_{2}$ move on $x$-axis and $q_{3}$ moves on $y$-axis. We denote

$$
q_{1}=(x(t), 0), q_{2}=(-x(t), 0), q_{3}=(0, y(t)) .
$$

Here $x(t)$ and $y(t)$ satisfy

$$
\begin{aligned}
& \ddot{x}=-\frac{1}{4 x^{2}} \\
& \ddot{y}=-\frac{2 y}{\left(x^{2}+y^{2}\right)^{3 / 2}} .
\end{aligned}
$$

By rescaling it, the equations become

$$
\begin{aligned}
& \ddot{x}=-\frac{1}{8 x^{2}} \\
& \ddot{y}=-\frac{y}{\left(x^{2}+y^{2}\right)^{3 / 2}} .
\end{aligned}
$$

Let $x(t)$ be the periodic solution of (2) with period 1 such that $x(t)=0(t \in \mathbb{Z})$ and $x(t)>0(t \in \mathbb{R} \backslash \mathbb{Z})$. The binary collision is regarded as regularized. The 
regularization can be intuitively regarded as the elastic bounce(Figure 2). See Appendix in detail.

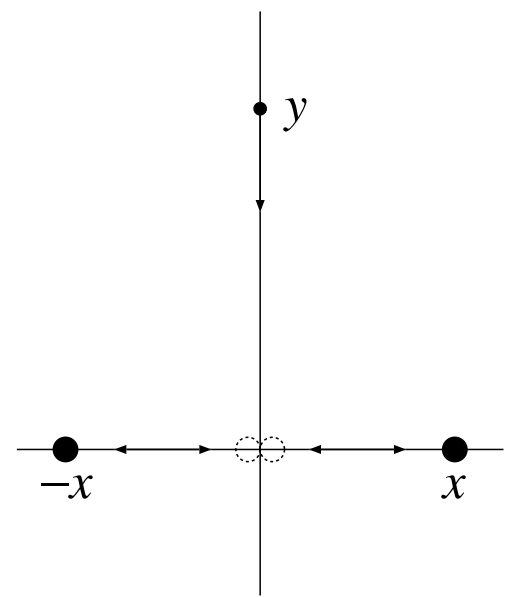

Figure 2: planar Sitnikov problem

Consider a sequence $\boldsymbol{a}=\left\{a_{n}\right\}_{n \in \mathbb{Z}} \in\{-1,1\}^{\mathbb{Z}}$. Define the subset $\mathcal{M}$ of $\{-1,1\}^{\mathbb{Z}}$ by the set of sequences $\boldsymbol{a}$ such that the length of each successive part of -1 or +1 is no less than 3 . More accurately, the sequence $\boldsymbol{a} \in\{-1,1\}^{\mathbb{Z}}$ is represented as

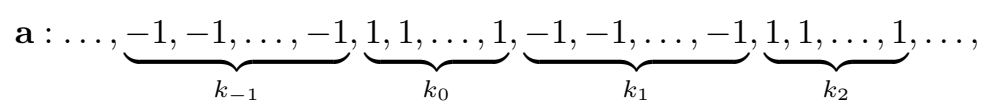

where $\left\{k_{j}\right\}_{j \in \Lambda} \subset \mathbb{N} \cup\{\infty\}$ may be finite, semi-finite or infinite sequence, that is, $\Lambda$ is the one of the following possiblity:

$$
\begin{aligned}
& \Lambda=\{I, I+1, I+2, \ldots, J-2, J-1, J\}, k_{I}=k_{J}=\infty \quad(I<J) \\
& \Lambda=\{I, I+1, I+2, \ldots\}, k_{I}=\infty \\
& (I \in \mathbb{Z}) \\
& \Lambda=\{\ldots, J-2, J-1, J\}, k_{J}=\infty \\
& (J \in \mathbb{Z}) \\
& \Lambda=\mathbb{Z} \text {. }
\end{aligned}
$$

Let $\mathcal{M}$ be the set of $\boldsymbol{a}$ such that $k_{j} \geq 3$ for any $j \in \Lambda$.

For $\boldsymbol{a} \in \mathcal{M}$ and for $N_{1}, N_{2} \in \mathbb{Z}\left(N_{1}<N_{2}\right)$, we define the set of functions

$\Omega_{N_{1}, N_{2}}(\mathbf{a})=\left\{y(t) \in H^{1}\left(\left[N_{1}, N_{2}\right], \mathbb{R}\right) \mid a_{n} \cdot y(n)>0\left(n=N_{1}, N_{1}+1, \ldots, N_{2}\right)\right\}$, $\Omega(\mathbf{a})=\left\{y(t) \in H_{\text {loc }}^{1}(\mathbb{R}, \mathbb{R}) \mid a_{n} \cdot y(n)>0(n \in \mathbb{Z})\right\}$

(See Figure 3).

Theorem 1. For any $\boldsymbol{a} \in \mathcal{M}, N_{1}, N_{2} \in \mathbb{Z}\left(N_{1}<N_{2}\right)$ and $\alpha, \beta>0$, there exists a solution $y(t) \in \Omega_{N_{1}, N_{2}}(\boldsymbol{a})$ of the planar Sitnikov problem such that 


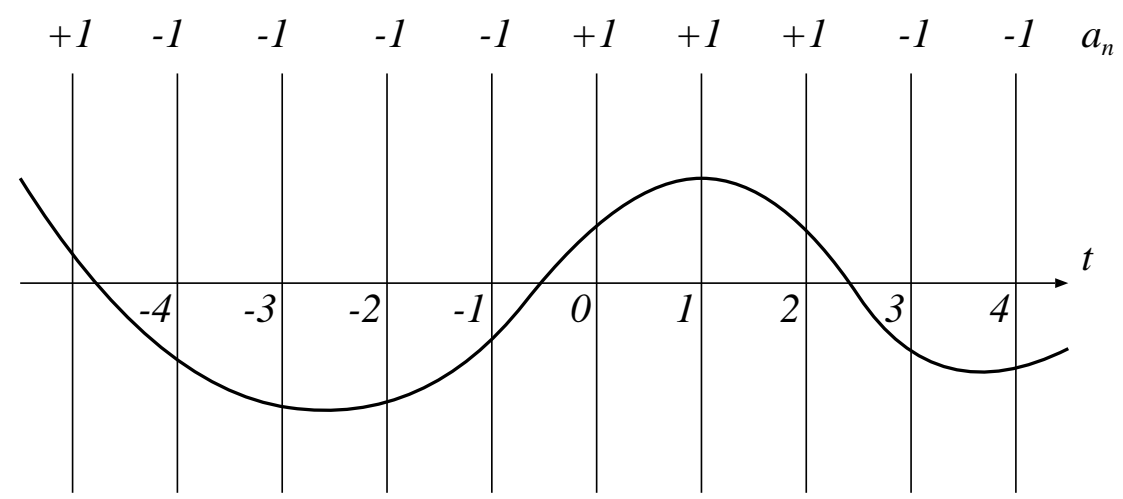

Figure 3: Behavior of $y(t) \in \Omega(\boldsymbol{a})$

$a_{N_{1}} y\left(N_{1}\right)=\alpha, a_{N_{2}} y\left(N_{2}\right)=\beta$. Moreover for $n \in\left\{N_{1}, N_{1}+1, \ldots, N_{2}-1\right\}$ satisfying $a_{n} a_{n+1}=1$, there is no real value $t \in(n, n+1)$ such that $y(t)=0$, and for $n \in\left\{N_{1}, N_{1}+1, \ldots, N_{2}-1\right\}$ satisfying $a_{n} a_{n+1}=-1$ there is a unique real number $t \in(n, n+1)$ such that $y(t)=0$.

There are two simple sequences $\boldsymbol{e}_{ \pm} \in \mathcal{M}$ such that

$$
\boldsymbol{e}_{+}=\ldots, 1,1,1, \ldots, \quad \boldsymbol{e}_{-}=\ldots,-1,-1,-1, \ldots
$$

For $N \in \mathbb{N}$, define $\mathcal{P}_{N}=\left\{\boldsymbol{a} \in \mathcal{M} \backslash\left\{\boldsymbol{e}_{ \pm}\right\} \mid a_{n}=a_{n+N}(n \in \mathbb{Z})\right\}$.

Theorem 2. For any $\boldsymbol{a} \in \mathcal{P}_{N}$, there exists an $N$-periodic solution $y(t) \in \Omega(\boldsymbol{a})$. Moreover for $n \in\{0,1, \ldots, N-1\}$ satisfying $a_{n} a_{n+1}=1$, there is no real value $t \in(n, n+1)$ such that $y(t)=0$, and for $n \in\{0,1, \ldots, N-1\}$ satisfying $a_{n} a_{n+1}=-1$ there is a unique real number $t \in(n, n+1)$ such that $y(t)=0$.

Remark 1. If $\boldsymbol{a} \in \mathcal{P}_{N}$, a also belongs to $\mathcal{P}_{k N}$ for $k \in \mathbb{N}$. We can obtain $k N$-periodic solution. But we do not know whether these periodic solutions are different.

This paper is organized as following. Next section, we introduce the variational formulation and prove the existence of generalized solutions. In Section 3 , we show that the generalized solutions have no collision and that they are classical solutions. In appendix, we illustrate how to regularize the binary collision.

\section{Variational formulation and the existence of generalized solutions}

We first show the existence of generalized solutions for Theorem 1. A generalized solution means a solution which is allowed to have triple collisions. Let $x(t)$ be 
determined as a solution with a collision per period 1. The equation (3) of $y$ is equivalent to the variational problem with respect to the action functional

$$
\mathcal{A}_{N_{1} N_{2}}(y)=\int_{N_{1}}^{N_{2}} L(y, \dot{y}, t) d t,
$$

where the Lagrangian is

$$
L(y, \dot{y}, t)=\frac{1}{2} \dot{y}^{2}+\frac{1}{\sqrt{(x(t))^{2}+y^{2}}} .
$$

We prove the coercivity of $\mathcal{A}_{N_{1}, N_{2}}(y)$ on

$$
\Gamma=\left\{y \in \Omega_{N_{1}, N_{2}}(\boldsymbol{a}) \mid y\left(N_{1}\right)=a_{N_{1}} \alpha, y\left(N_{2}\right)=a_{N_{2}} \beta\right\}
$$

for Theorem 1 . We can assume that $N_{1}=0, N_{2}=N$ without loss of generality. Let $|y(t)|(t \in[0, N])$ attain the maximum $\max _{t \in[0, N]}|y(t)|$ at $t_{\max }$, and let $\|\cdot\|_{L^{2}}$ and $\langle\cdot, \cdot\rangle_{L^{2}}$ be the $L^{2}$-norm and -inner product. We estimate the $L^{2}$-norm of $y$ in the set (4) as follows:

$$
\begin{aligned}
\|y\|_{L^{2}}^{2} & =\int_{0}^{N} y^{2} d t \leq N\left|y\left(t_{\max }\right)\right|^{2}=2 N\left|\int_{0}^{t_{\max }} \dot{y}(t) d t+a_{0} \alpha\right|^{2} \\
& \leq 2 N\left(\left|\int_{0}^{t_{\max }} \dot{y}(t) d t\right|+|\alpha|\right)^{2} \leq 2 N\left(\int_{0}^{t_{\max }}|\dot{y}(t)| d t+|\alpha|\right)^{2} \\
& \leq 2 N\left(\int_{0}^{N}|\dot{y}(t)| d t+|\alpha|\right)^{2}=2 N\left(\|\dot{y}\|_{L^{1}}+|\alpha|\right)^{2}
\end{aligned}
$$

where $\|\cdot\|_{L^{1}}$ is the $L^{1}$-norm. Since

$$
\|\dot{y}\|_{L^{1}}^{2}=\langle 1,|\dot{y}|\rangle_{L^{2}}^{2} \leq\|1\|_{L^{2}}^{2}\|\dot{y}\|_{L^{2}}^{2}=N\|\dot{y}\|_{L^{2}}^{2},
$$

$\|\dot{y}\|_{L^{2}}$ diverses to infinity as $\|y\|_{L^{2}} \rightarrow \infty$. Therefore since

$$
\|\dot{y}\|_{L^{2}}^{2} \leq 2 \mathcal{A}_{N}(y),
$$

$\mathcal{A}_{N}(y)$ diverses to infinity as $\|y\|_{H^{1}} \rightarrow \infty$ for $y$ in the set (4). This means that $\mathcal{A}_{N}$ restricted on the set is coercive, and hence there exists a minimizer in the closure $\bar{\Omega}_{N_{1} N_{2}}$ of $\Omega_{N_{1} N_{2}}$.

Now we shall prove of the existence for Theorem 2. For each $y \in \Omega(\boldsymbol{a})$, there is $t_{0}$ such that $y\left(t_{0}\right)=0$ since $\boldsymbol{a} \neq \boldsymbol{e}_{ \pm}$. Let $\max _{t \in[0, N]}|y(t)|$ be attained at $t_{\max }$ and similarly we get

$$
\begin{aligned}
\|y\|_{L^{2}}^{2} & \leq \int_{0}^{N} y^{2} d t \leq N\left|y\left(t_{\max }\right)\right|^{2}=2 N\left|\int_{t_{0}}^{t_{\max }} \dot{y}(t) d t\right|^{2} \\
& \leq 2 N\left(\int_{t_{0}}^{t_{\max }}|\dot{y}(t)| d t\right)^{2} \leq 2 N\left(\int_{0}^{N}|\dot{y}(t)| d t\right)^{2} \\
& \leq 2 N\left(\int_{0}^{N}|\dot{y}(t)| d t\right)^{2}=2 N\|\dot{y}\|_{L^{1}}^{2} .
\end{aligned}
$$


The rest of the proof is the same as one for Theorem 1 .

\section{The number of $t$ with $y(t)=0$ on $(n, n+1)$}

We estimate the number of $t$ satisfying $f(t)=0$ on $(n, n+1)$. When $y(t)$ minimizes $\mathcal{A}_{N_{1} N_{2}}$, we can assume that $n=0$ and $y$ minimizes $\mathcal{A}_{0,1}$. Suppose that $y$ minimizes $\mathcal{A}_{0,1}$. We show that the number of $t \in(0,1)$ such that $y(t)=0$ is at most one or $y(t)$ is identically zero on $(0,1)$.

Assume that there is $t_{0} \in(0,1)$ such that $y\left(t_{0}\right)=0$. If $\dot{y}\left(t_{0}\right)=0, y(t)$ and $-y(t)$ are solutions with the same initial condition. From the uniqueness of the solution of ODE, $y(t)$ is identically zero.

Assume that there are two $t \in(0,1)$ such that $y(t)=0$ but that $y(t)$ is not identically zero. Let $y\left(t_{0}\right)=y\left(t_{1}\right)=0\left(0<t_{0}<t_{1}<1\right)$. Since $y(t)$ is not identically zero, $\dot{y}\left(t_{0}\right) \neq 0, \dot{y}\left(t_{1}\right) \neq 0$. Define a partial modified path by

$$
\tilde{y}(t)= \begin{cases}y(t) & \left(t \in\left[0, t_{0}\right] \cup\left[t_{1}, 1\right]\right) \\ -y(t) & \left(t \in\left(t_{0}, t_{1}\right)\right)\end{cases}
$$

This path $\tilde{y}$ also minimizes $\mathcal{A}_{0,1}$, but $\tilde{y}$ is not smooth. This contradicts the fact that the minimizer is smooth.

\section{Exclusion of triple collisions}

\subsection{Sundmann estimate}

We eliminate the value of the action functional with a triple collision. We show it for Theorem 1 and 2 simultaneously. Assume that $y(0)=0$ (and assume that $N_{1}<0<N_{2}$ for Theorem 1). For any minimizer $y,\left.y\right|_{[-1,1]}$ minimizes $\mathcal{A}_{-1,1}$. From the Sundman estimate, the asymptotic behavior as $t \rightarrow \pm 0$ can be represented by

$$
x(t)=c|t|^{2 / 3}+O(t), \quad y_{N}(t)=d_{ \pm}|t|^{2 / 3}+O(t)
$$

where $c=2^{-4 / 3} \cdot 3^{2 / 3}$ and $d_{ \pm} \in\left\{-2^{-4 / 3} \cdot 3^{7 / 6}, 0,2^{-4 / 3} \cdot 3^{7 / 6}\right\}$. We make a modified curve with a lower value of the action functional.

Note that $d_{+}=0$ if and only if $y(t)$ is identically zero on $(0,1)$. It can be seen from the collision manifold theory. The unstable manifold corresponding to the configuration is one dimensional. McGehee [3] has first constructed such coordinates for the collinear three-body problem, and Devaney [2] has applied it to the isosceles three-body problem. Meyer and Wang [4] have studied the restricted case of the the isosceles three-body problem which means $m_{3}=0$. That is the same as what we are considering. 
The value of the integral on the interval $[0, \varepsilon]$ is

$$
\begin{aligned}
\mathcal{A}_{0, \varepsilon}(y) & =\int_{0}^{\varepsilon} \frac{1}{2}\left(\frac{2}{3} d_{+} t^{-1 / 3}+O(1)\right)^{2}+\frac{1}{\sqrt{\left(c t^{2 / 3}+O(t)\right)^{2}+\left(d_{+} t^{2 / 3}+O(t)\right)^{2}}} d t \\
& =\int_{0}^{\varepsilon}\left(\frac{2}{9} d_{+}^{2}+\frac{1}{\sqrt{c^{2}+d_{+}^{2}}}\right) t^{-2 / 3}+O\left(t^{-1 / 3}\right) d t \\
& =\left[3\left(\frac{2}{9} d_{+}^{2}+\frac{1}{\sqrt{c^{2}+d_{+}^{2}}}\right) t^{1 / 3}+O\left(t^{2 / 3}\right)\right]_{0}^{\varepsilon} \\
& =\left(\frac{2}{3} d_{+}^{2}+\frac{3}{\sqrt{c^{2}+d_{+}^{2}}}\right) \varepsilon^{1 / 3}+O\left(\varepsilon^{2 / 3}\right)
\end{aligned}
$$

The estimate of $\mathcal{A}_{-\varepsilon, 0}$ is similar, and hence we get

$$
\mathcal{A}_{-\varepsilon, \varepsilon}=\left(\frac{2}{3}\left(d_{+}^{2}+d_{-}^{2}\right)+\frac{3}{\sqrt{c^{2}+d_{+}^{2}}}+\frac{3}{\sqrt{c^{2}+d_{-}^{2}}}\right) \varepsilon^{1 / 3}+O\left(\varepsilon^{2 / 3}\right) .
$$

We will make modified curves with a less value for each case.

We can assume $a_{0}=1$ without loss of generality since we can replace $\left\{-a_{n}\right\}$ if $a_{0}=-1$. Since $\boldsymbol{a} \in \mathcal{M}$, there are two cases: the both sides are same as $a_{0}$ i.e. $a_{-1}=a_{0}=a_{1}=1$; one of the sides is different from $a_{0}$ i. e. $a_{-1}=-1, a_{0}=a_{1}=1$ or $a_{-1}=a_{0}=1, a_{1}=-1$ whose latter case is essentially same as the former one. Hence we need to consider the case of $a_{-1}=a_{0}=a_{1}=1$ and $a_{-1}=-1, a_{0}=a_{1}=1$.

\subsection{The case of $a_{-1}=a_{0}=a_{1}=1$}

In this case, $y(-1) \geq 0$ and $y(1) \geq 0$. If $y(-1)>0, d_{-}=0$ or $d_{-}=2^{-4 / 3} \cdot 3^{7 / 6}$ from Section 3. If $y(-1)=0$ and $y(t)<0(-\varepsilon<t<0)$, we can replace $y(t)$ by $-y(t)$. Hence we need to consider the case of $d_{-}=0$ or $d_{-}=2^{-4 / 3} \cdot 3^{7 / 6}$. Therefore it is sufficient to study 4 different case determined by $d_{ \pm} \in\left\{0,2^{-4 / 3}\right.$. $\left.3^{7 / 6}\right\}$.

The case of $d_{+}=d_{-}=2^{-4 / 3} \cdot 3^{7 / 6} \quad$ Define a modified path for $t \in(-\varepsilon, \varepsilon)$ by

$$
\begin{aligned}
y_{\text {test }}(t) & =\frac{1}{2 \varepsilon}(y(\varepsilon)-y(-\varepsilon))(t+\varepsilon)+y(-\varepsilon) \\
& =d_{-} \varepsilon^{2 / 3}+O(\varepsilon) .
\end{aligned}
$$

We get

$$
\dot{y}_{\text {test }}(t)=O(1)
$$


Hence we have

$$
\begin{aligned}
\mathcal{A}_{-\varepsilon, \varepsilon}\left(y_{\text {test }}\right) & =\int_{-\varepsilon}^{\varepsilon} O(1)+\frac{1}{d_{-} \varepsilon^{2 / 3}} d t=\frac{2}{d_{-}} \varepsilon^{1 / 3}+O(\varepsilon)=2^{-5 / 3} \cdot 3^{-1 / 6} \varepsilon^{1 / 3}+O(\varepsilon) \\
& \approx 1.398820085 \varepsilon^{1 / 3}+O(\varepsilon) .
\end{aligned}
$$

On the other hand, (5) is

$$
2^{-2 / 3} \cdot 3^{1 / 3} \cdot 7 \varepsilon^{1 / 3}+O(\varepsilon)=6.359922076 \varepsilon^{1 / 3}+O(\varepsilon) .
$$

The case of $d_{+}=2^{-4 / 3} \cdot 3^{7 / 6}, d_{-}=0 \quad$ Define a modified path for $t \in(-\varepsilon, \varepsilon)$ by

$$
\begin{aligned}
y_{\text {test }}(t) & =\frac{1}{2 \varepsilon}(y(\varepsilon)-y(-\varepsilon))(t+\varepsilon)+y(-\varepsilon) \\
& =\frac{1}{2} d_{+} \varepsilon^{-1 / 3}(t+\varepsilon)+O(\varepsilon) .
\end{aligned}
$$

We get

$$
\dot{y}_{\text {test }}(t)=\frac{1}{2} d_{+} \varepsilon^{-1 / 3}+O(1)
$$

Since $0<y_{\text {col }}(t)<y_{\text {test }}(t)$,

$$
\int_{-\varepsilon}^{\varepsilon} \frac{1}{\sqrt{x(t)^{2}+y_{\mathrm{col}}(t)^{2}}} d t \geq \int_{-\varepsilon}^{\varepsilon} \frac{1}{\sqrt{x(t)^{2}+y_{\text {test }}(t)^{2}}} d t .
$$

The kinetic part of the test path is

$$
\begin{aligned}
\int_{-\varepsilon}^{\varepsilon} \frac{1}{2}\left(\frac{1}{2} d_{+} \varepsilon^{-1 / 3}+O(1)\right)^{2} d t & =\frac{1}{4} d_{+}^{2} \varepsilon^{1 / 3}+O\left(\varepsilon^{2 / 3}\right)=2^{-14 / 3} \cdot 3^{7 / 3} \varepsilon^{1 / 3}+O\left(\varepsilon^{2 / 3}\right) \\
& \approx 0.5110651668 \varepsilon^{1 / 3}+O\left(\varepsilon^{2 / 3}\right)
\end{aligned}
$$

and one of the collision path is

$$
\begin{aligned}
\frac{2}{3}\left(d_{+}^{2}+d_{-}^{2}\right) \varepsilon^{1 / 3}+O\left(\varepsilon^{2 / 3}\right) & =2^{-5 / 3} \cdot 3^{4 / 3} \varepsilon^{1 / 3}+O\left(\varepsilon^{2 / 3}\right) \\
& \approx 1.362840445 \varepsilon^{1 / 3}+O\left(\varepsilon^{2 / 3}\right) .
\end{aligned}
$$

Then by summing them, the test path has lower value of the action functional.

The case of $d_{+}=0, d_{-}=2^{-4 / 3} \cdot 3^{7 / 6} \quad$ Similar as the previous case.

The case of $d_{+}=d_{-}=0 \quad$ Define a test path by

$$
y_{\text {test }}(t)=\varepsilon^{2 / 3}-|t|^{2 / 3} \quad t \in(-\varepsilon, \varepsilon) .
$$


The kinetic part is

$$
\int_{-\varepsilon}^{\varepsilon} \frac{1}{2} \dot{y}^{2}=\int_{0}^{\varepsilon}\left(\frac{2}{3} t^{-1 / 3}\right)^{2} d t=\frac{4}{9} \cdot 3\left[t^{1 / 3}\right]_{0}^{\varepsilon}=\frac{4}{3} \varepsilon^{1 / 3}
$$

Since

$$
\begin{aligned}
y_{\text {test }}(t)^{2}+x(t)^{2} & =\left(\varepsilon^{2 / 3}-|t|^{2 / 3}\right)^{2}+c^{2}|t|^{4 / 3}+O\left(|t|^{5 / 3}\right) \\
& =\left(c^{2}+1\right)\left(|t|^{4 / 3}-\frac{\varepsilon^{2 / 3}}{c^{2}+1}\right)^{2}+\frac{c^{2} \varepsilon^{4 / 3}}{c^{2}+1}+O\left(\varepsilon^{5 / 3}\right),
\end{aligned}
$$

the potential part is estimated as follows:

$$
\int_{-\varepsilon}^{\varepsilon} \frac{1}{\sqrt{y_{\text {test }}(t)^{2}+x(t)^{2}}} d t \leq \frac{2 \sqrt{c^{2}+1} \varepsilon^{1 / 3}}{c}+O\left(\varepsilon^{2 / 3}\right) .
$$

The value of the action functional is estimated

$$
\mathcal{A}_{-\varepsilon, \varepsilon}\left(y_{\text {test }}\right)=2\left(\frac{2}{3}+\frac{\sqrt{c^{2}+1}}{c}\right) \varepsilon^{1 / 3}+O\left(\varepsilon^{2 / 3}\right) \approx 4.475003732 \varepsilon^{1 / 3}+O\left(\varepsilon^{2 / 3}\right)
$$

This value is lower than one for collision paths because

$$
\int_{-\varepsilon}^{\varepsilon} \frac{1}{c t^{2 / 3}} d t=\frac{6 \varepsilon^{1 / 3}}{c} \approx 7.268482372 \varepsilon^{1 / 3}
$$

\subsection{The case of $a_{-1}=-1, a_{0}=a_{1}=1$}

Take a modified path as follows

$$
y_{\text {test }}= \begin{cases}-(y(-\varepsilon)-\delta)(\varepsilon-k)^{-1}(t+k)+\delta & t \in[-\varepsilon,-k] \\ \delta & t \in[-k, 0] \\ (y(\varepsilon)-\delta) \varepsilon^{-1} t+\delta & t \in[0, \varepsilon]\end{cases}
$$

where $\delta=l \varepsilon^{2 / 3}, k=\varepsilon a$ and $l, a \in(0,1)$ is some constant.

The integral on interval $[0, \varepsilon]$ is estimated as follows:

$$
\begin{aligned}
\mathcal{A}_{0, \varepsilon}\left(y_{\text {test }}\right) & =\int_{0}^{\varepsilon} \frac{1}{2}(y(\varepsilon)-\delta)^{2} \varepsilon^{-2}+\frac{1}{\sqrt{\left(\left(c t^{2 / 3}+O(t)\right)^{2}+\left((y(\varepsilon)-\delta) \varepsilon^{-1} t+\delta\right)^{2}\right.}} d t \\
& \geq \frac{1}{2}(y(\varepsilon)-\delta)^{2} \varepsilon^{-1}+\varepsilon \delta^{-1} \\
& =\frac{1}{2}\left(d_{+} \varepsilon^{2 / 3}+O(\varepsilon)-\delta\right)^{2} \varepsilon^{-1}+\varepsilon \delta^{-1} \\
& =\left(\frac{1}{2}\left(d_{+}-l\right)^{2}+l^{-1}\right) \varepsilon^{1 / 3}+O(\varepsilon) .
\end{aligned}
$$

The integral on interval $[-k, 0]$ is

$$
\mathcal{A}_{-k, 0}\left(y_{\text {test }}\right) \geq \frac{k}{\delta}=a l^{-1} \varepsilon^{1 / 3} .
$$


The integral on interval $[-\varepsilon,-k]$ is

$$
\begin{aligned}
\mathcal{A}_{-\varepsilon,-k}\left(y_{\text {test }}\right) & \geq\left(\frac{1}{2}(y(-\varepsilon)-\delta)^{2}(\varepsilon-k)^{-1}+3 c^{-1}\left[|t|^{1 / 3}\right]_{-\varepsilon}^{-k}\right)+O(\varepsilon) \\
& =\left(\frac{1}{2}\left(d_{-} \varepsilon^{2 / 3}-l \varepsilon^{2 / 3}\right)^{2}(\varepsilon-k)^{-1}+3 c^{-1}\left(\varepsilon^{1 / 3}-k^{1 / 3}\right)\right)+O(\varepsilon) \\
& =\left(\frac{1}{2}\left(d_{-} \varepsilon^{2 / 3}-l \varepsilon^{2 / 3}\right)^{2}(1-a)^{-1} \varepsilon^{-1}+3 c^{-1}\left(1-a^{1 / 3}\right) \varepsilon^{1 / 3}\right)+O(\varepsilon) \\
& =\left(\frac{1}{2}\left(d_{-}-l\right)^{2}(1-a)^{-1}+3 c^{-1}\left(1-a^{1 / 3}\right)\right) \varepsilon^{1 / 3}+O(\varepsilon) .
\end{aligned}
$$

The value of the integral $[-\varepsilon, \varepsilon]$ is

$$
\begin{aligned}
\mathcal{A}\left(y_{\text {test }}\right) & \leq\left(\left(\frac{1}{2}\left(d_{+}-l\right)^{2}+l^{-1}\right)+a l^{-1}+\left(\frac{1}{2}\left(d_{-}-l\right)^{2}(1-a)^{-1}+3 c^{-1}\left(1-a^{1 / 3}\right)\right)\right) \varepsilon^{1 / 3}+O\left(\varepsilon^{2 / 3}\right) \\
& =\frac{1}{2}\left(d_{+}-l\right)^{2}+(a+1) l^{-1}+\frac{1}{2}\left(d_{-}-l\right)^{2}(1-a)^{-1}+3 c^{-1}\left(1-a^{1 / 3}\right) \\
& =\frac{1}{2}\left(d_{+}-d s\right)^{2}+(a+1) d^{-1} s^{-1}+\frac{1}{2}\left(d_{-}+d s\right)^{2}(1-a)^{-1}+3 c^{-1}\left(1-a^{1 / 3}\right) \\
& =\frac{d^{2}}{2}(1-s)^{2}+(a+1) d^{-1} s^{-1}+\frac{d^{2}}{2}(1+s)^{2}(1-a)^{-1}+3 c^{-1}\left(1-a^{1 / 3}\right) \\
& =\left(\frac{d_{+}^{2}}{2}\left((1-s)^{2}+(1+s)^{2}(1-a)^{-1}\right)+(a+1) d^{-1} s^{-1}+3 c^{-1}\left(1-a^{1 / 3}\right)\right) \varepsilon^{1 / 3}+O\left(\varepsilon^{2 / 3}\right)
\end{aligned}
$$

By taking $a=0.2, s=0.5$,

$$
\left(\frac{d_{+}^{2}}{2}\left((1-s)^{2}+(1+s)^{2}(1-a)^{-1}\right)+(a+1) d^{-1} s^{-1}+3 c^{-1}\left(1-a^{1 / 3}\right)\right) \approx 6.317782296
$$

and the value for collision paths is 6.359922076 .

\section{The number of $t$ with $y(t)=0$ on $(n, n+1)$ (revised)}

As we showed, the set of $t \in(n, n+1)$ satisfying $y(t)=0$ is empty set, one point or $(n, n+1)$. Since triple collisions do not occur, the third case is impossible. If $a_{n} a_{n+1}=-1$, it is one point. If $a_{n} a_{n+1}=1$, it is empty set from the uniqueness of the solution.

\section{A Appendix:Regularization of collision}

Here we introduce regularization of the binary collision in the planar Sitnikov problem. The planar Sitnikov problem is equivalent to one-order differential 
equations:

$$
\begin{aligned}
\frac{d x}{d t} & =p_{x} \\
\frac{d p_{x}}{d t} & =-\frac{1}{8 x^{2}} \\
\frac{d y}{d t} & =p_{y} \\
\frac{d p_{y}}{d t} & =-\frac{y}{\left(x^{2}+y^{2}\right)^{3 / 2}} .
\end{aligned}
$$

Now we regularize the singularity $x=0$. By using the Levi-Civita transform $x=\frac{1}{2} \xi^{2}, p_{x}=p_{\xi} / \xi$, we get

$$
\begin{aligned}
\frac{d \xi}{d t} & =\frac{p_{\xi}}{\xi^{2}} \\
\frac{d p_{\xi}}{d t} & =\frac{p_{\xi}^{2}}{\xi^{3}}-\frac{1}{2 \xi^{3}} \\
\frac{d y}{d t} & =p_{y} \\
\frac{d p_{y}}{d t} & =-\frac{y}{\left(\frac{\xi^{4}}{4}+y^{2}\right)^{3 / 2}} .
\end{aligned}
$$

The motion of $m_{1}$ and $m_{2}$ conserves the energy

$$
E=\frac{1}{2} p_{x}^{2}-\frac{1}{8 x}=\frac{1}{\xi^{2}}\left(\frac{1}{2} p_{\xi}^{2}-\frac{1}{4}\right) .
$$

By using the energy relation, the equations become

$$
\begin{aligned}
\frac{d \xi}{d t} & =\frac{p_{\xi}}{\xi^{2}} \\
\frac{d p_{\xi}}{d t} & =\frac{2 E}{\xi} \\
\frac{d y}{d t} & =p_{y} \\
\frac{d p_{y}}{d t} & =-\frac{y}{\left(\frac{\xi^{4}}{4}+y^{2}\right)^{3 / 2}} .
\end{aligned}
$$

Finally by changing time variable from $t$ to $s$ according to $\frac{d s}{d t}=\frac{1}{\xi^{2}}$, we obtain 
the following equations:

$$
\begin{aligned}
\frac{d \xi}{d t} & =p_{\xi} \\
\frac{d p_{\xi}}{d t} & =2 E \xi \\
\frac{d y}{d t} & =p_{y} \xi^{2} \\
\frac{d p_{y}}{d t} & =-\frac{y \xi^{2}}{\left(\frac{\xi^{4}}{4}+y^{2}\right)^{3 / 2}} .
\end{aligned}
$$

The singularity $x=0$ which corresponds to $\xi=0$ is regularized. Here we fix $E<0$ so that the period is one in the original time. Remark that the Levi-Civita transform $\left(\xi, p_{\xi}\right) \mapsto\left(x, p_{x}\right)$ is two to one. $\left(\left(\xi, p_{\xi}\right)\right.$ and $\left(-\xi,-p_{\xi}\right)$ corresponds to the same $\left.\left(x, p_{x}\right)\right)$.

Acknowledgement The author is supported by the Japan Society for the Promotion of Science (JSPS), Grant-in-Aid for Scientific Research (C), 18K03366.

\section{References}

[1] A. Chenciner and R. Montgomery, A remarkable periodic solution of the three-body problem in the case of equal masses Ann. Math. 152(2000), 881901.

[2] R. L. Devaney, Triple collision in the planar isosceles three-body problem. Invent. Math. 60 (1980), 249-267.

[3] R. McGehee, Triple collision in the collinear three-body problem. Invent. Math. 27 (1974), 191-227.

[4] K. Meyer \& Q. D. Wang, Global phase structure of the restricted isosceles three-body problem with positive energy. Trans. Amer. Math. Soc. 338 (1993), 311-336.

[5] K. Sitnikov, The existence of oscillatory motions in the three-body problems. Dokl. Akad. Nauk SSSR 133, pp. 303-306 (Russian); translated as Soviet Physics. Dokl. 5 (1960), pp. 647-650. 